\title{
HIGHLIGHTS
}

\section{Trimodal therapy a promising alternative for high-risk patients}

The outcomes of a combined regimen of hormone therapy, external beam radiation therapy (EBRT) and brachytherapy compare favorably with those of standard treatments in men with prostate cancer who have a Gleason score of $8-10$. This three-pronged approach is "better able to control disease compared to ... radical prostatectomy or EBRT alone" asserts lead author Richard Stock from the Mount Sinai School of Medicine, New York.

The trimodal regimen takes advantage of two important advances in radiation therapy for prostate cancer-concomitant hormone treatment and dose escalation. In Stock et al.'s 181-strong series, patients with locally advanced disease received neoadjuvant hormone therapy for 3 months before implantation of a low-dose (100 Gy) brachytherapy device. Starting 2 months later, 3-dimensional conformal and intensity-modulated techniques were used to deliver $251.8 \mathrm{~Gy}$ fractions of EBRT, together with a further 6 months of hormone therapy. The mean biologically effective dose
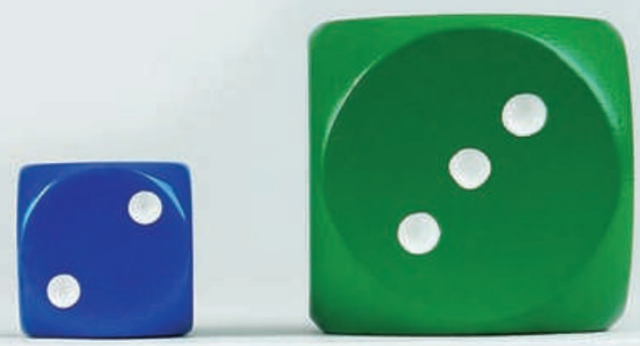

generated by brachytherapy plus EBRT was very high, at $206 \mathrm{~Gy}^{2}$.

Follow-up at 6-month intervals continued for a median of 65 months. At 8 years, overall rates of freedom from biochemical failure, freedom from distant metastasis, prostate-cancer-specific survival and overall survival were $73 \%$, $80 \%, 87 \%$ and $79 \%$, respectively.

Gleason score was the best predictor of response to treatment. The poorer outcomes among the subset of men with a Gleason score of $10-70 \%$ biochemical failure, $70 \%$ distant metastases, and $63 \%$ prostate cancer-specific survivalnecessitate development of "better systemic approaches to combine with local therapy".

\section{Rebecca Drake}

Original article Stock, R. G. et al. Outcomes for patients with high-grade prostate cancer treated with a combination of brachytherapy, external beam radiotherapy and hormonal therapy. BJU Int. 104, 1631-1636 (2009) 